\title{
Carbamazepine Induced Stevens-Johnson Syndrome (SJS)
}

\author{
Sourya Acharya ${ }^{1}$, Vidyashree Hulkoti², Amol Andhale ${ }^{3}$, Sugat Jawade ${ }^{4}$, Sneha Deshmukh ${ }^{5}$
}

1,2, 3,5 Department of Medicine, Datta Meghe Institute of Medical Sciences (DMIMS), (Deemed to Be University), Sawangi (Meghe), Wardha, Maharashtra, India. ${ }^{4}$ Department of Dermatology, Datta Meghe Institute of Medical Sciences (DMIMS), (Deemed to Be University), Sawangi (Meghe), Wardha, Maharashtra, India.

\section{INTRODUCTION}

Stevens-Johnson syndrome (SJS) and toxic epidermal necrolysis (TEN), are dermatologic emergencies characterised by a blistering rash that appears in a variable percentage of the body-surface. The mortality risk increases with increasing epidermal detachment. TEN is the rarest of these phenotypes and is associated with mortality of up to $30 \% .{ }^{1}$ Exposure to certain drugs, some infections, contrast media, and vaccines can predispose to development of SJS. We report a case of carbamazepine induced SJS.

\section{PRESENTATION OF CASE}

A 35-year-old male, chronic alcoholic presented to us with a generalised symmetrical, erythematosus rash, which was fast spreading and affected the face, upper limbs, chest and had breathlessness of 3 days duration. He had been drinking alcohol daily for last 3 months. Because of some problem he didn't get alcohol for 3 days and had a status generalised tonic clonic seizure for which he was taken to a private hospital where he received IV lorazepam and carbamazepine $200 \mathrm{mg}$ t.d.s. per orally. He developed dyspnoea and skin rash 3 days after oral carbamazepine.

On examination, facial swelling was present; bullae were present on neck and chest; there were ulcerations in the mouth associated with conjunctival injection and difficulty in opening eyes. Pulse - 118 / min, temperature $-39.2^{\circ} \mathrm{C}$, with $\mathrm{SpO}_{2}$ of $87 \%$ in ambient air, and blood pressure of 92 / $50 \mathrm{mmHg}$. Vesiculobullous lesions developed over the course of the admission period which was associated with sloughing of skin on the chest, face and lips (Fig. 1,2).

Investigations: $\mathrm{CBC}-\mathrm{Hb}-9.2$ gram \%, TLC- 2,500 / mm 3, Neutrophils- $88 \%$. Liver and renal function was normal. A diagnosis of carbamazepine induced StevenJohnson syndrome was made. Evaluation of Toxic Epidermal Necrosis (SCORTEN) score on admission was 2. CXR (chest X-ray) PA revealed right lower lobe consolidation.

\author{
Corresponding Author: \\ Dr. Sourya Acharya, \\ Professor, Department of Medicine, \\ Datta Meghe Institute of Medical \\ Sciences (DMIMS), (Deemed to Be \\ University), Sawangi (Meghe), \\ Wardha, Maharashtra, India. \\ E-mail: souryaacharya74@gmail.com
}

DOI: $10.14260 /$ jemds/2020/852

How to Cite This Article: Acharya S, Hulkoti V, Andhale A, et al. Carbamazepine induced stevens-Johnson syndrome (SJS). J Evolution Med Dent Sci 2020;9(51):3892-3893, $10.14260 /$ jemds/2020/852 DOI:

Submission 07-04-2020,

Peer Review 28-10-2020,

Acceptance 04-11-2020,

Published 21-12-2020.

Copyright (C) 2020 Sourya Acharya et al. This is an open access article distributed under Creative Commons Attribution License [Attribution 4.0 International (CC BY 4.0)] 

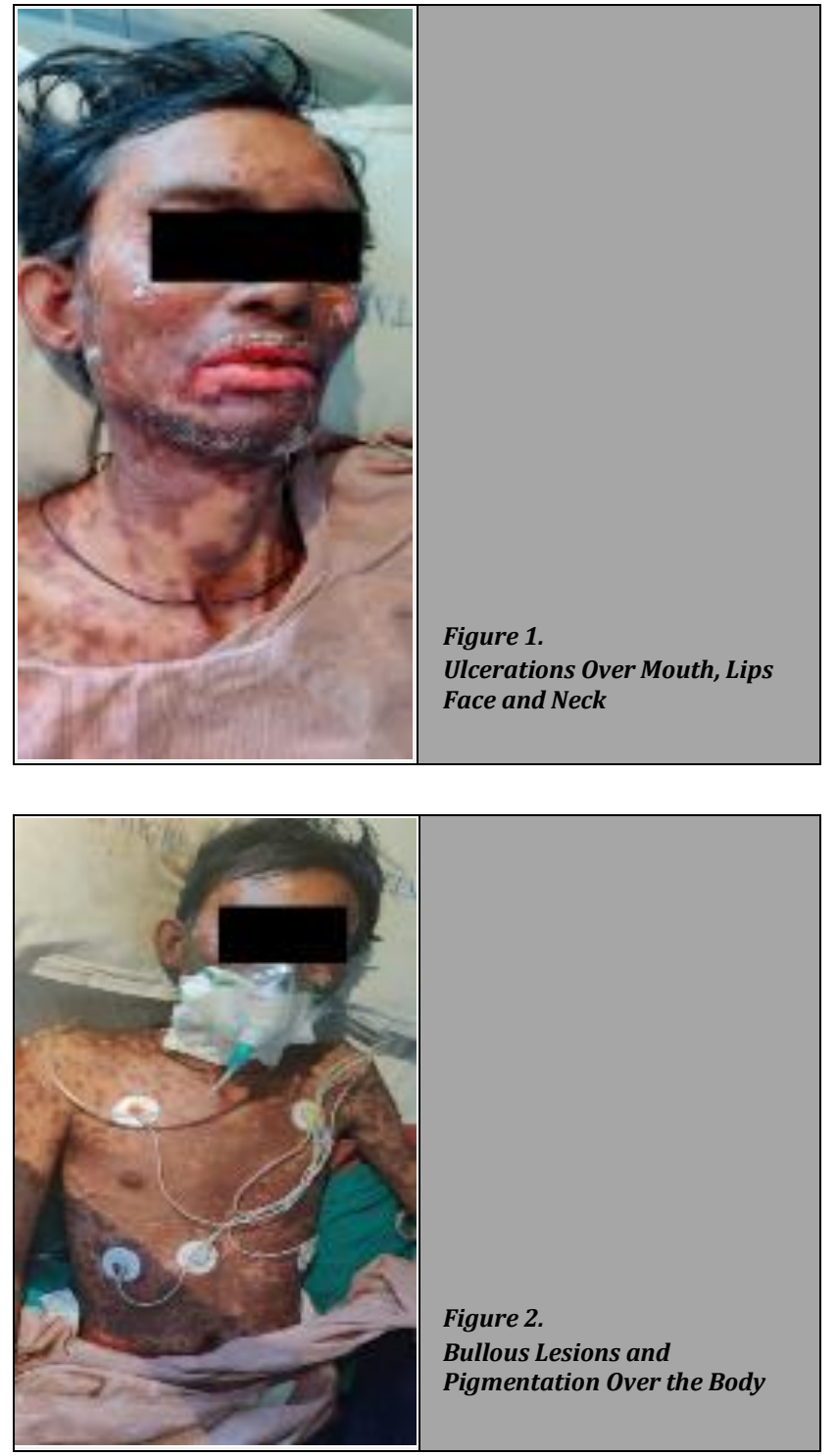

DISCUSSION OF MANAGEMENT

Carbamazepine was discontinued and the patient was treated with intravenous fluids, IV antibiotics, IV dexamethasone, and antiseptic mouth wash. The patient recovered in 2 weeks.

\section{DISCUSSION}

Anti-epileptic drugs (AEDs) that can cause SJS / TEN are phenytoin, phenobarbital, lamotrigine, and carbamazepine. ${ }^{2}$ Two to five percent of patients receiving AED develop cutaneous adverse drug reactions (CADRs). ${ }^{3}$ According to a study conducted by Lange-Asschenfeldt et al. phenytoin, phenobarbital, lamotrigine, and carbamazepine are the most common AEDs that develop SJS. Carbamazepine is a wellknown causative agent of drug induced SJS among antiepileptic drugs. ${ }^{3}$

SJS is an unpredictable adverse effect of carbamazepine. It is usually idiosyncratic and dose independent. In Asian and Caucasian populations carbamazepine associated SJS is linked to HLA-B (human leukocyte antigen) * 15:02 and HLA-B * 31:01.4 We could not perform genotype study in our patient for HLA alleles. Other factors that can play adjuvant role in development of SJS / TEN due to carbamazepine are, individual variability in drug metabolism, HIV (Human Immunodeficiency Virus) infection, polypharmacy, and competitive drug inhibition. ${ }^{5}$

Financial or other competing interests: None.

Disclosure forms provided by the authors are available with the full text of this article at jemds.com.

\section{REFERENCES}

[1] McCormack M, Alfirevic A, Bourgeois S, et al. HLA - A*3101 and carbamazepine - induced hypersensitivity reactions in Europeans. N Engl J Med 2011;364(12):1134-43.

[2] Dodiuk-Gad RP, Laws PM, Shear NH. Epidemiology of severe drug hypersensitivity. Semin Cutan Med Surg 2014;33(1):2-9.

[3] Lange-Asschenfeldt C, Grohmann R, Lange-Asschenfeldt $\mathrm{B}$, et al. Cutaneous adverse reactions to psychotropic drugs: data from a multicenter surveillance program. J Clin Psychiatry 2009;70(9):1258-65.

[4] Amstutz U, Shear NH, Rieder MJ, et al. Recommendations for HLA-B*15:02 and HLA-A*31:01 genetic testing to reduce the risk of carbamazepine-induced hypersensitivity reactions. Epilepsia 2014;55(4):496506.

[5] Uetrecht J, Naisbitt DJ. Idiosyncratic adverse drug reactions: current concepts. Pharmacol Rev 2013;65(2):779-808. 